\title{
Malestar emocional en los familiares de adolescentes con asma bronquial: análisis de sus predictores
} Emotional distress in family caregivers of adolescents with bronchial asthma: analysis of its predictors

\author{
Psicóloga Selene Valero-Moreno ${ }^{a}$, Dra. Marián Pérez-Marin ${ }^{b}$, \\ Dra. Inmaculada Montoya-Castillab , Dra. Silvia Castillo-Corullónc, \\ Dr. Álvar Ramírez-Aguilarc y Dra. Amparo Escribano-Montanerc,d
}

a. Facultad de

Psicología.

Universitat de

València.

b. Departamento de Personalidad, Evaluación y Tratamientos Psicológicos, Facultad de Psicología. Universitat de València.

c. Unidad de Neumología Infantil y Fibrosis Quística. Hospital Clínico y Universitario de Valencia.

d. Departamento de Pediatría, Obstetricia y Ginecología, Facultad de

Medicina,

Universitat de València

Valencia, España.

Correspondencia: Dra. Marián Pérez-Marín: marian.perez@uv.es

Financiamiento:

El estudio ha sido realizado gracias a las ayudas de investigación recibidas por la Asociación Española de Psicología Clínica y Psicopatología y por la Federación Española de Asociaciones de Terapia Familiar.

Conflicto de intereses: Ninguno que declarar.

Recibido: 26-7-2017

Aceptado: 19-9-2017

\section{RESUMEN}

Introducción. Los adolescentes asmáticos se enfrentan a problemas propios de la etapa a los que se añaden asumir el control de su enfermedad, el cumplimiento de un tratamiento diario y de los controles médicos periódicos. El posible rechazo que esto genere puede conducir a la no adherencia terapéutica y al mal control del asma, lo que ocasiona problemas en la dinámica familiar, agravados por el estrés o el malestar emocional que esta situación provoca en los cuidadores.

Objetivo. Identificar perfiles de adaptación y detectar predictores de riesgo para el bienestar emocional de los cuidadores de pacientes pediátricos con asma bronquial.

Material y métodos. 79 cuidadores familiares de pacientes pediátricos con asma bronquial. Se utilizan instrumentos para evaluar la clínica psicológica emocional (Hospital Anxiety and Depression, HADS) y el nivel de estrés percibido asociado al cuidado médico de un paciente pediátrico (Pediatric Inventory for Parents, PIP). Se analiza el papel de las variables médicas del paciente y las psicológicas del cuidador familiar relativas a su malestar emocional y estrés.

Resultados. 34,8\% de los cuidadores presenta sintomatología ansiosa y estrés percibido moderado. La sintomatología emocional se relaciona positiva y significativamente con el nivel de estrés. Los modelos que mejor predicen el malestar emocional de los cuidadores (40$50 \%$ de varianza explicada) incluyen como predictores indicadores de estrés, tiempo desde el diagnóstico y carga terapéutica del paciente. Conclusiones. La presencia de estrés derivado del cuidado, el tiempo de exposición y la carga de los tratamientos asociados a la enfermedad se identificaron como los principales predictores de riesgos para el bienestar del cuidador.

Palabrasclave: asmabronquial, adolescente, familiar cuidador, bienestar emocional, adaptación psicológica.

http:/ / dx.doi.org/10.5546/aap.2018.e234

Texto completo en inglés:

http: / / dx.doi.org/10.5546/ aap.2018.eng.e234

Cómo citar: Valero-Moreno S, Pérez-Marín M, Montoya-Castilla I, et al. Malestar emocional en los familiares de adolescentes con asma bronquial: análisis de sus predictores. Arch Argent Pediatr 2018; 116(2):e234-e240.

\section{INTRODUCCIÓN}

El asma es la enfermedad crónica más prevalente en la infancia y adolescencia. ${ }^{1}$ En España, el 5-14\% de los adolescentes, de 13-14 años, sufren asma. ${ }^{2,3} \mathrm{La}$ adolescencia es, por sí misma, un período de grandes cambios biopsicosociales. El adolescente asmático, además de los hitos normativos de esta etapa evolutiva, ha de atender los tratamientos y efectos de su enfermedad, lo que puede ser una dificultad añadida en la construcción de su propia identidad, la asunción de responsabilidades, el logro de independencia de su sistema familiar de referencia, el desarrollo de su imagen corporal, la maduración de su personalidad e, incluso, en la relación con su grupo de iguales. ${ }^{4}$

Entre los problemas más frecuentes referidos por los profesionales de la salud que atienden a adolescentes asmáticos está el cambio de actitud que sufren frente a la enfermedad, que puede pasar de una postura amable, más dependiente y colaborativa a otra más hostil, defensiva o negativista. Esto puede condicionar su manejo y autocontrol, y poner en riesgo el buen curso de la enfermedad. ${ }^{5} \mathrm{La}$ falta de adherencia al tratamiento puede deberse, entre otras razones, al desconocimiento de la enfermedad y de la importancia de mantener el tratamiento, así como de las consecuencias de no hacerlo, pero, también, al olvido no intencionado de la medicación, al rechazo activo de las prescripciones médicas, a la minimización de la necesidad de 
tratamiento en períodos asintomáticos o a la relativización de los posibles efectos secundarios. ${ }^{6}$

La literatura científica subraya la importancia del apoyo familiar para un buen ajuste al proceso de enfermedad en este tipo de pacientes, que es una situación estresante para todo el sistema familiar. Los cuidadores familiares de adolescentes que requieren seguimiento médico prolongado suelen presentar síntomas de ansiedad ${ }^{7,8}$ y clínica depresiva ${ }^{9-11}$ con más frecuencia y con niveles más elevados que los que refieren los padres de adolescentes sanos. ${ }^{12,13} \mathrm{~A}$ su vez, los pacientes con pobre supervisión parental tienen mayores complicaciones y peor control de la enfermedad. ${ }^{14}$

El objetivo de nuestro estudio es identificar perfiles de adaptación y riesgo para el bienestar emocional de los cuidadores familiares de pacientes pediátricos con asma bronquial.

\section{MATERIAL Y MÉTODOS Participantes}

Muestra constituida por cuidadores familiares principales de pacientes pediátricos (de 9-18 años de edad), en seguimiento durante 2016, en la Unidad de Neumología Pediátrica del Hospital Clínico Universitario de Valencia (HUCV) y que firmaron el consentimiento informado para participar en el estudio.

Los criterios de inclusión en el estudio fueron cuidador principal familiar (aquel que asumía en mayor medida el cuidado habitual y hospitalario del paciente, al menos, 15 horas semanales) de pacientes de 9-18 años de edad, que presentaban asma bronquial diagnosticada, al menos, 6 meses antes y controlada bajo tratamiento en los últimos 6 meses. El diagnóstico de asma y su control se estableció según los criterios de la Guía Española de Manejo del Asma (GEMA). ${ }^{2}$

Los criterios de exclusión en el estudio fueron cuidadores de pacientes con alguna de las siguientes enfermedades subyacentes: a) parálisis cerebral infantil, b) tumor cerebral, c) diagnóstico psicológico previo al inicio de la enfermedad respiratoria.

\section{Instrumentos utilizados}

Pediatric Inventory for Parents (PIP), ${ }^{15,16}$ cuestionario de 42 ítems diseñado para evaluar los niveles de estrés percibido por los familiares ante el cuidado de una enfermedad crónica pediátrica. Se basa en el modelo transaccional de Lazarus y Folkman, ${ }^{17}$ que considera el estrés como un proceso que se pone en marcha cuando la persona percibe una situación externa como amenazante y que desborda los recursos que posee para afrontarla. Esto exige, en ocasiones, un sobreesfuerzo que puede poner en peligro el bienestar personal. ${ }^{17}$ Cada ítem plantea una situación de cuidado ante la que se tiene en cuenta lo siguiente: 1) la frecuencia de aparición (de nunca a muy frecuente) y 2) el nivel de esfuerzo psicológico percibido que genera en el cuidador (de nada a muchísimo). El cuestionario ofrece dos puntuaciones totales relacionadas con los dos indicadores globales de estrés y, además, proporciona información relativa a 4 factores: cuidados médicos, comunicación, estrés emocional y rol familiar. El PIP ha mostrado, en diversas investigaciones, cualidades psicométricas adecuadas. $^{15}$

Hospital Anxiety and Depression (HADS), ${ }^{18}$ instrumento de screening para detectar la sintomatología ansioso-depresiva, especialmente, cognitiva, en el medio hospitalario no psiquiátrico o en atención primaria. La exclusión de la medición de síntomas somáticos (insomnio, fatiga, pérdida de apetito, etc.) es muy importante, pues evita equívocos de atribución en personas con algún tipo de enfermedad, debido al posible solapamiento en los síntomas médicos y psicológicos en estos casos. La subescala de depresión está centrada en el concepto de anhedonia como síntoma nuclear de este cuadro clínico y que diferencia primordialmente la ansiedad de la depresión. ${ }^{19}$ Se divide en dos escalas que evalúan los principales elementos de la clínica afectiva: presencia de síntomas de ansiedad (7 ítems) y de depresión (7 ítems). Recoge también una medida global de esta clínica de malestar emocional. Estudios sobre sus propiedades psicométricas en la población española han mostrado valores adecuados. ${ }^{20}$

\section{Procedimiento}

Diseño transversal, con un pase de evaluación único, en un solo momento temporal, el día de visita de seguimiento del paciente a la consulta de Neumología Pediátrica. Un psicólogo entrenado explicó las características y fines del estudio al cuidador familiar, solicitó su aceptación para participar y la firma del consentimiento informado, que aseguró la confidencialidad. El propio cuidador cumplimentó los cuestionarios.

Los resultados se analizaron en relación con los siguientes parámetros clínicos: cronicidad de la enfermedad (al menos, 6 meses desde el diagnóstico), gravedad y estado de control 
del asma (criterios según la GEMA), presencia -o no- de otras enfermedades respiratorias (especialmente, alergia como diagnóstico secundario). Se recogió el tratamiento diario utilizado (con el número de dosis de inhaladores (aerosoles), terapia nebulizada, medicación oral y/o inmunoterapia) para observar la carga que podría suponer este tratamiento en los cuidadores.

El trabajo contó con el aval de los Comités de Ética de las entidades participantes en el estudio (Universidad de Valencia y Fundación INCLIVA - Instituto de Investigación Sanitaria del Hospital Clínico Universitario de Valencia-).

Los datos se analizaron con el programa estadístico SPSS 22.0.

\section{RESULTADOS}

Se evaluaron 79 cuidadores familiares principales de pacientes asmáticos; $77,2 \%$ eran madres $(n=61)$ y $22,8 \%$, padres $(n=18)$, con una edad comprendida entre los 35 y los 54 años (media $=44,66$; desviación típica - D.T. $-=4,68$; mediana $=45)$.

\section{Variables médicas de los pacientes}

Todos los pacientes presentaban asma bronquial clínicamente controlada, al menos, desde los últimos 6 meses, independientemente del tratamiento médico recibido. Los pacientes se clasificaban según los criterios de gravedad de la GEMA en el momento de inclusión (Tabla 1). Aun así, un 17,7\% ( $\mathrm{n}=14)$ presentaba una inflamación eosinofílica no controlada [óxido nítrico exhalado $(\mathrm{ONe}) \geq 30 \mathrm{ppb}$. Se analizaron también las

TABLA 1. Gravedad del asma en función de la Guía Española de Manejo del Asma y comorbilidades con otros diagnósticos

\begin{tabular}{lcc}
\hline Gravedad del asma & $\mathbf{n}$ & $\%$ \\
\hline Episódico-ocasional & 2 & 2,5 \\
Episódico-frecuente & 14 & 17,7 \\
Persistente-moderado & 62 & 78,5 \\
Persistente-grave & 1 & 1,3 \\
Comorbilidades & & \\
& $\mathbf{n}$ & $\%$ \\
\hline Asma bronquial & 17 & 21,5 \\
Asma bronquial más otro diagnóstico & 53 & 67,1 \\
Asma bronquial más dos diagnósticos & 6 & 7,6 \\
Asma bronquial más tres diagnósticos & 2 & 2,5 \\
Asma bronquial más cuatro diagnósticos & 1 & 1,3 \\
\hline
\end{tabular}

n: frecuencia de casos; \%: porcentaje total de casos de determinada categoría. comorbilidades (Tabla 1), y se destacó la alergia a inhalantes $(69,6 \%)$, clínicamente controlada en la mayoría de los casos $(75,4 \%)$. Respecto al grupo de malacias (traqueomalacia, broncomalacia, laringomalacia), estas solamente mostraron comorbilidad con el asma $(3,8 \%)$, pero no con otros diagnósticos médicos.

El tiempo medio de cronicidad de la enfermedad desde su diagnóstico fue alrededor de los 5 años (Tabla 2) y las dosis medias de tratamiento recibido eran de 1,86/ día (datos más completos del tratamiento médico y la frecuencia de visitas se muestran en la Tabla 3).

\section{Análisis descriptivos de las variables clínicas del cuidador}

Un tercio $(33,3 \%)$ de los familiares mostró sintomatología ansiosa (sensación de angustia/temor, preocupación mantenida e hiperactivación); un 14,1\% presentó un posible diagnóstico de trastorno de ansiedad. Cerca del 13\% mostró síntomas depresivos (anhedonia, autoconcepto negativo, pérdida de interés por su imagen) y, en un $3,8 \%$, estos eran lo suficientemente llamativos como para indicar la presencia de un trastorno del estado de ánimo (Tabla 4). Respecto al malestar emocional global, el 5,1\% mostraba indicadores del diagnóstico de un trastorno emocional.

Tal como se recoge en la Tabla 5, los cuidadores familiares presentan un estrés moderado en las diferentes subescalas del PIP, en las que se destacan las puntuaciones en frecuencia de aparición de las situaciones de cuidado. Entre las situaciones de cuidado que generan estrés, sobresalen las de la escala de estrés emocional (relacionadas con malestar emocional, incertidumbre y preocupación por la enfermedad, tanto en uno mismo como en el hijo).

Tabla 2. Estadísticos descriptivos de variables médicas: tiempo de cronicidad y dosis diarias

\begin{tabular}{lccc}
\hline & & $\begin{array}{c}\text { Tiempo desde } \\
\text { el diagnóstico } \\
\text { (en meses) }\end{array}$ & $\begin{array}{c}\text { Dosis diaria } \\
\text { total }\end{array}$ \\
\hline $\mathrm{n}$ & Válido & 70 & 79 \\
$\mathrm{X}$ & Perdidos & 9 & 0 \\
Mediana & 70,63 & 1,86 & \\
$\mathrm{DT}$ & 57,00 & 2,00 & \\
Mín. & 57,378 & 1,195 & \\
Máx. & 6 & 0 & \\
\hline
\end{tabular}

n: frecuencia de casos; X: media; DT: desviación típica. 


\section{Análisis de correlación y predicción de las variables}

Como se puede observar en la Tabla 6, ansiedad, depresión y malestar emocional global se correlacionan positivamente con el estrés experimentado por el cuidador. Así, en los dos indicadores globales de estrés; 1) frecuencia de aparición de las situaciones de cuidado y 2) esfuerzo psicológico asociado a ellas, las puntuaciones más altas en estrés se asocian con valores mayores en los tres indicadores de clínica psicológica emocional (ansiedad, depresión y malestar emocional global).

En particular, cabe señalar que la ansiedad y el malestar emocional global están especialmente relacionados con la alta frecuencia de aparición de situaciones de cuidado en la escala de estrés emocional.

Se analizó también la relación existente entre las variables médicas (tiempo de cronicidad, número de dosis diarias utilizadas, comorbilidades, gravedad del asma y control de la alergia) y las de clínica psicológica emocional (ansiedad, depresión y malestar emocional global). Tras el análisis, en nuestros datos, no aparecen relaciones estadísticamente significativas entre ambos grupos de variables.

Por último, se llevaron a cabo tres análisis

TABLA 3. Tipo de tratamiento médico utilizado y frecuencia de visitas de los adolescentes asmáticos

\begin{tabular}{lcc}
\hline Tipo de tratamiento & $\mathbf{n}$ & $\%$ \\
\hline Terapia inhalada con aerosoles o polvo seco & 43 & 54,4 \\
Terapia inhalada con aerosoles o polvo seco y medicación oral & 26 & 32,9 \\
Terapia inhalada con aerosoles o polvo seco e inmunoterapia & 7 & 8,9 \\
Terapia inhalada con aerosoles o polvo seco, medicación oral e inmunoterapia & 3 & 3,8 \\
\hline Frecuencia de visitas & $\mathbf{n}$ & $\%$ \\
\hline Anual & 57 & 72,2 \\
Cada 6 meses & 26 & 32,9 \\
Cada 4 meses & 5 & 6,3 \\
Cada 3 meses & 1 & 1,3 \\
\hline
\end{tabular}

n: frecuencia de casos; \%: porcentaje total de casos de determinada categoría.

TABLA 4. Indicadores de malestar emocional: clínica ansioso-depresiva

\begin{tabular}{lccccccc}
\hline & \multicolumn{2}{c}{ Normal-ausencia } & \multicolumn{2}{c}{ Caso probable } & \multicolumn{3}{c}{ Problema clínico } \\
& $\mathbf{n}$ & $\%$ & $\mathbf{N}$ & $\%$ & \multicolumn{2}{c}{ n } & $\%$ \\
\hline Ansiedad & 52 & 66,7 & 15 & 19,2 & 11 & 14,1 \\
Depresión & 68 & 87,2 & 7 & 9 & 3 & 3,8 \\
Malestar emocional global & 74 & 94,9 & - & - & 4 & 5,1 \\
\hline
\end{tabular}

n: frecuencia de casos; \%: porcentaje total de casos de determinada categoría.

TABLA 5. Indicadores del estrés percibido ante las tareas de cuidado de un paciente con asma

\begin{tabular}{lccccc}
\hline & M & DT & Mín. & Máx. & $\begin{array}{c}\text { Rango de puntuaciones } \\
\text { de las escalas }\end{array}$ \\
\hline 1. Comunicación Frecuencia & 20,65 & 4,06 & 12 & 31 & $9-45$ \\
1. Comunicación Esfuerzo & 16,61 & 5,45 & 9 & 36 & $9-45$ \\
2. Cuidados médicos Frecuencia & 24,29 & 5,23 & 11 & 36 & $8-40$ \\
2. Cuidados médicos Esfuerzo & 14,49 & 5,60 & 8 & 32 & $8-40$ \\
3. Estrés emocional Frecuencia & 35,51 & 8,02 & 20 & 67 & $15-75$ \\
3. Estrés emocional Esfuerzo & 37,13 & 11,35 & 16 & 70 & $15-75$ \\
4. Rol familiar Frecuencia & 21,34 & 5,53 & 10 & 41 & $10-50$ \\
4. Rol familiar Esfuerzo & 21,46 & 7,05 & 10 & 45 & $10-50$ \\
5. Total Frecuencia & 101,34 & 19,1 & 57 & 90 & $42-210$ \\
6. Total Esfuerzo & 89,56 & 25,64 & 44 & 183 & $42-210$
\end{tabular}

M: media; DT: desviación típica; Mín.: puntuación mínima obtenida en el cuestionario; Máx.: puntuación máxima que se puede obtener en el cuestionario. 
de regresión lineal múltiple para estudiar qué variables podían ayudarnos a predecir mejor las puntuaciones en los tres indicadores de clínica psicológica emocional (ansiedad, depresión, malestar emocional global). Inicialmente, se incluyeron como posibles variables predictoras las siguientes: a) los dos indicadores globales de estrés y b) las variables médicas estudiadas.

El modelo que mejor nos ayuda a predecir el "nivel de malestar emocional global experimentado por el cuidador familiar" incluye como variables predictoras "número de dosis diarias de tratamiento médico recibido" y los dos indicadores globales de estrés: 1) frecuencia de aparición de las situaciones de cuidado y 2) esfuerzo psicológico asociado a ellas. Este modelo explica el 37,3\% de la varianza. Tras realizar un análisis de regresión conglomerado, se encontró que el tipo de situaciones que mejor predecía los niveles de estrés ligado a la frecuencia de aparición de situaciones de cuidado sería el incluido en los factores estrés emocional (malestar emocional, incertidumbre y preocupación por la enfermedad) y cuidados médicos (someterse a pruebas médicas, cambios en el tratamiento o toma de decisiones sobre cuestiones médicas). Respecto al nivel de estrés ligado al esfuerzo psicológico asociado al cuidado, el análisis reveló que eran las situaciones incluidas en el factor rol familiar (cambios de rol familiar, laboral o social) las que, principalmente, influían en este tipo de estrés experimentado por los cuidadores.

El modelo que mejor predice el "nivel de ansiedad del cuidador familiar" incluye como variables predictoras "nivel de depresión en el cuidador", "indicador global de estrés ligado a la frecuencia de aparición de las situaciones de cuidado" y "tiempo de cronicidad", que explica el 47,8\% de la varianza. En el análisis de regresión conglomerado realizado, se encontró que el tipo de situaciones que mejor predecía los niveles de estrés relacionados con la frecuencia de aparición de situaciones ligadas al cuidado sería el incluido en el factor estrés emocional.

Por último, el modelo que mejor explica el "nivel de depresión experimentado por el familiar" es el que incluye las variables predictoras "nivel de ansiedad en el cuidador", "indicador global de estrés ligado al esfuerzo psicológico asociado a las situaciones de cuidado" y "dosis diarias de los tratamientos médicos recibidos", que explica el $48,6 \%$ de la varianza. Respecto al nivel de estrés ligado al esfuerzo psicológico asociado al cuidado, las situaciones incluidas en los factores cuidados médicos y rol familiar (situaciones estresantes relacionadas con cambios de rol a nivel familiar, laboral o social) serían las que, principalmente, influirían en este tipo de estrés experimentado por los cuidadores.

\section{DISCUSIÓN}

Los resultados señalan que un elevado número de cuidadores de adolescentes asmáticos (incluso con asma clínicamente controlada) muestra niveles importantes de estrés y sintomatología ansiosa-depresiva, que ocasionan un impacto negativo en su bienestar y aumentan el riesgo de padecer algún trastorno emocional.

Tabla 6. Asociaciones entre las variables médicas del paciente y clinicas del cuidador

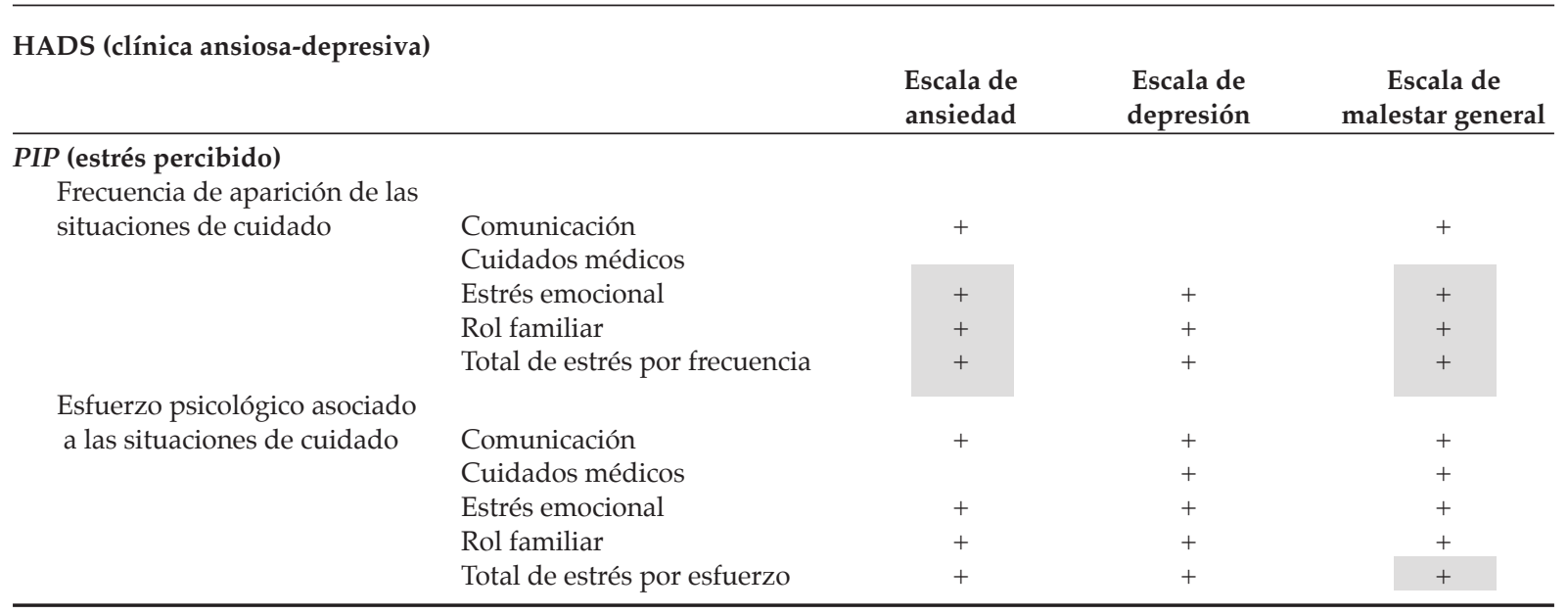

Relación moderada: color blanco; relación alta o fuerte: color gris.

HADS: Hospital Anxiety and Depression; PIP: Pediatric Inventory for Parents. 
En los estudios, se han observado resultados similares en cuidadores de pacientes con talla baja $^{7}$ o con diabetes, ${ }^{8}$ en los que se detectan niveles moderados de estrés, con las puntuaciones más elevadas en las subescalas de cuidados médicos y estrés emocional. Así, el sistema familiar de un paciente pediátrico con asma debe enfrentarse, además de a los estresores comunes a todas las familias con hijos adolescentes, a los originados por la presencia de la enfermedad. Estos factores adicionales de estrés incluyen aspectos como los siguientes: ${ }^{15,16}$ a) la propia enfermedad en sí y la novedad e incertidumbre sobre su curso y evolución; b) la comprensión de la información que brinda el personal sanitario, explicaciones sobre la enfermedad al adolescente y al entorno más cercano, el tener que abordar la confrontación o resistencia del niño a su tratamiento; c) las dificultades económicas, al tener que dedicar parte del presupuesto familiar a tratar la enfermedad (que repercute en otras necesidades, como ocio, recreación o compartir experiencias); d) el manejo psicoemocional de los elementos ligados a los cuidados médicos necesarios y e) las tensiones con la pareja y familia, y conflictos de roles.

En nuestro estudio, un elevado porcentaje de los cuidadores de pacientes asmáticos presentó sintomatología ansiosa y depresiva, aspecto señalado también en estudios realizados en cuidadores de niños con talla baja, ${ }^{7}$ diabetes mellitus tipo $1^{8}$ o cáncer, ${ }^{10}$ en los que, en un porcentaje importante de casos, estos presentaban un problema clínico que requería atención psicológica.

Nuestra investigación tiene algunas limitaciones, como que la gran mayoría de cuidadores eran mujeres, dado que suelen ser las madres las que se dedican, principalmente, al cuidado de sus hijos. Podría ser interesante observar las posibles diferencias en el ajuste a la enfermedad entre los padres y madres de los adolescentes enfermos. Además, con el objetivo de homogenizar la muestra, nuestro estudio solo se ha centrado en pacientes con asma bronquial clínicamente controlado. Futuras investigaciones podrían evaluar la relación existente entre el grado de control del asma y la repercusión emocional en los cuidadores principales, así como con otras variables médicas, como el número de hospitalizaciones y su duración, o las exacerbaciones.

Un abordaje multidisciplinar del asma bronquial en la adolescencia permitiría ir más allá de la propia patología médica y conocer aspectos que podrían interferir en el adecuado controlevolución de la enfermedad. Así, se pondrían en marcha actuaciones concretas dirigidas a prevenir la psicopatología en el conjunto del sistema familiar y, con ello, mejorar su calidad de vida.

\section{CONCLUSIONES}

El 33,3\% de los cuidadores mostraba síntomas ansiosos y el 13\%, depresivos. Se identificaron como principales predictores de riesgo de malestar emocional los niveles de estrés del familiar asociados a las conductas de cuidado junto con el tiempo transcurrido desde el diagnóstico de los pacientes pediátricos y la carga terapéutica a la que estos se debían enfrentar diariamente.

\section{REFERENCIAS}

1. Organización Mundial de la Salud. Enfermedades respiratorias crónicas. 2016. [Acceso: 1 de junio de 2017]. Disponible en: http: / / www.who.int/ respiratory / asthma/es/.

2. GEMA. Guía Española para el Manejo del Asma. 2016. [Acceso: 15 deabrilde2017]. Disponibleen: www.gemasma. com.

3. European Respiratory Society European Lung White Book: Respiratory Health and Disease in Europe. 2013. [Acceso: 1 de junio de 2017]. Disponible en: http: / / www. erswhitebook.org/.

4. Pellegrini-Belinchón J, de Arriba Méndez S. El asma en el adolescente. Adolescere 2013;1(3):7-27.

5. Alonso Lebrero E. Asma en situaciones especiales. En García-Marcos Álvarez L, Garde J, Escribano Montaner A, et al. Asma en pediatría. Barcelona: Edipharma; 2002. Págs.187-217.

6. Martín Martín R. Estudio de las variables psicológicas, calidad de vida e intervención psicoeducativa en el asma bronquial infantil [Tesis]. Salamanca: Universidad de Salamanca; 2008.

7. Casaña-GranellS, Lacomba-Trejo L, Montoya-Castilla I, et al. Malestar emocional y estrés en cuidadores principales de pacientes pediátricos con talla baja. Calid Vida Salud 2016;9(2):93-103.

8. Lacomba-Trejo L, Casaña-Granell S, Pérez-Marín M, et al. Estrés, Ansiedad y Depresión en cuidadores principales de pacientes pediátricos con Diabetes Mellitus Tipo 1. Calid Vida Salud 2017;10(1):10-22.

9. Pak L, Allen PJ. The impact of maternal depression on children with asthma. Pediatr Nurs 2012;38(1):11-30.

10. Rodríguez E, Dunn M, Zuckerman T, et al. Cancer-related sources of stress for children with cancer and their parents. J Pediatr Psychol 2012;37(2):185-97.

11. Blanco Ávila DM. Habilidad de cuidado de los cuidadores principales de niños en situación de enfermedad crónica que consultan en la Clínica Colsanitas. Av Enferm 2007;25(1):19-32.

12. Bazán Riverón GE. Evaluación del impacto familiar del asma bronquial infantil en población mexicana. [Tesis]. Barcelona: Universidad Autónoma de Barcelona; 2009.

13. González-Barcala JF, García-Couceiro N, Facal D. Educación en asma. Arch Bronconeumol 2016;52(11):543-4. 
e240 / Arch Argent Pediatr 2018;116(2):e234-e240 / Artículo original

14. Mann NM. Compliance among adolescents with asthma. [Tesis]. Utah: Universidad de Utah, 2000.

15. Streisand R, Braniecki S, Teryack K, et al. Childhood Illness-Related Parenting Stress: The Pediatric Inventory for Parents. J Pediatr Psychol 2001;26(3):155-62.

16. Del Rincón C, Remor E, Arranz P. Estudio psicométrico preliminar de la versión española del Pediatric Inventory for Parents (PIP). Int J Clin Health Psychol 2007;7(2):435-52.
17. Lazarus RS, Folkman S. Estrés y procesos cognitivos. Barcelona: Martínez Roca; 1986.

18. Zigmond AS, Snaith RP. The hospital anxiety and depression scale. Acta Psychiatr Scand 1983;67(6):361-70.

19. American Psychiatric Association. DSM-5. Diagnostic and Statistical Manual of Mental Disorders. 5. ${ }^{a}$ ed. Arlington, VA: American Psychiatric Publishing; 2014.

20. Gabalda IC, Ibañez E. La escala hospitalaria de ansiedad y depresión. Bol Psicol 1992;36:43-69. 